\title{
Saberes compartidos entre América Latina y la Organización Internacional del Trabajo: un recuento historiográfico contemporáneo
}

\author{
Knowledge shared between Latin America and the International Labor \\ Organization: a contemporary historiographical account
}

\author{
Patricio Herrera González* \\ Universidad de Valparaíso (UV), Valparaíso, Chile \\ Juan Carlos Yáñez Andrade* \\ Universidad de Valparaíso (UV), Valparaíso, Chile
}

\begin{abstract}
RESUMEN: A propósito del centenerio de la Organización Internacional del Trabajo (OIT), este artículo establece un recuento historiográfico y temático que viene a reafirmar que América Latina y la OIT construyeron en conjunto un campo de saberes técnicos y políticos, lo cual permite proporcionar una nueva interpretación de una historia global o conectada, por sobre las fronteras nacionales, y privilegiando una escala regional. A contracorriente de la propia OIT y sus historiadoras/es oficiales del centenario, que siguen reconstruyendo una historia con mirada eurocéntrica, esta contribución da cuenta que América Latina, a través del impulso de liderazgos políticos, sindicales, empresariales e intelectuales, le concedió a la organización ginebrina de presentarse como un actor posible para intentar cumplir su misión, de garantizar la paz universal y establecer justicia social.
\end{abstract}

PALABRAS CLAVE: América Latina. OIT. Historiografía. Saberes. Sociolaboral.

\begin{abstract}
Regarding the centenary of the International Labor Organization (ILO), this article establishes a historical and thematic account that reaffirms that Latin America and the ILO jointly built a field of technical and political knowledge, which allows for a new interpretation of a global or connected history, over national borders, and privileging a regional scale. Contrary to the ILO itself and its official centenary historians, who continue to reconstruct a history with a Eurocentric gaze, this account contribution that Latin America, through the encouragement of political leaders, unions, businessmen and intellectuals, granted to the Geneva organization to present yourself as a possible actor to try to fulfill your mission, to determine universal peace and establish social justice.
\end{abstract}

KEYWORDS: Latin America. ILO. Historiography. Knowledge. Social and labor.

\footnotetext{
* Profesor de la Facultad de Ciencias Económicas y Administrativas de la Universidad de Valparaíso e investigador del CIDEP. Doctor en Historia. E-mail: patricio.herrera@uv.cl. https://orcid.org/0000-0001-9776-1911

* Profesor de la Facultad de Ciencias Económicas y Administrativas de la Universidad de Valparaíso e investigador del CIDEP. Doctor en Historia. E-mail: juancarlos.yanez@uv.cl. https://orcid.org/0000-0003-0317-3292
} 


\section{Introducción}

Esta contribución hace un recuento de los estudios sociolaborales y de colaboración entre América Latina, particularmente del sur, y la Organización Internacional del Trabajo (OIT), cuestión que ha tenido una creciente ocupación por historiadoras/es y cientistas sociales en la última década, a propósito del centenario de la organización ginebrina, pero también en una búsqueda por explorar otras interpretaciones de la historia social, política e institucional de nuestro continente, como una manera de vincular procesos nacionales a escala regional e internacional, complementando así respuestas que en las historiografías se conciben desde la evolución política local/nacional y contingente.

La apertura de archivos nacionales e internacionales, el acceso a documentos de manera remota en diversas bibliotecas y la multiplicación de formas de hacer historia, transnacional, conectadas, interdisciplnarias, permitió en las dos últimas décadas avanzar en nuevas interpretaciones sobre las representaciones e identidades, circulaciones y experiencias vinculadas a la OIT y America Latina.

A pesar de que América Latina estuvo presente con funcionarios desde muy temprano en la organización internacional de Ginebra (HERRERA LEÓN; HERRERA GONZÁLEZ, 2013), y que Albert Thomas, primer director de la OIT, visitó el continente en 1925 (FERRERAS, 2011; YÁNEZ, 2016; FERRERAS, 2019), esto no fue consignado por las historiografías del continente sino hasta hace unas décadas. La OIT, al igual que otros organismos internacionales, fue considerada por investigadores y ensayistas de la región como instancias desprovistas de instrumentos para evaluar y empatizar con los problemas reales del continente, asociadas a una diplomacia de funcionarios de escritorio y conferencias, que establecían resoluciones de papel y sin incidencia en la vida de trabajadoras/es, organizaciones obreras, gremios de la industria, en materias tan importantes como legislación laboral y seguridad social.

Si bien el aporte de Moisés Poblete Troncoso, abogado chileno, experto en derecho social y económico y funcionario de carrera en la Oficina Internacional del Trabajo de Ginebra, puede considerarse pionero en establecer la trascendencia de la OIT para el derecho laboral y social del continente y sus múltiples efectos positivos para los trabajadores, empresariado y Estado. Hay que reconocer también que su perspectiva jurídica, institucional y unidireccional generó limitaciones a sus interpretaciones, aunque sus numerosas publicaciones dejan una herencia inconmensurable (HERRERA GONZÁLEZ, 2013b; YÁÑEZ, 2017; 2018)ํㅜ y son fuente inagotable para seguir avanzando en reconstruir una historia que fue compleja, donde las aportaciones técnicas, sindicales, legislativas, productivas y de gobernanza fueron una aprendizaje de ida y vuelta, tanto para la naciones latinoamericanas como para la OIT. Esta última, tuvo que expandir sus conocimientos de problemas laborales y sociales, distintos a los estudiados y legislados en Europa. Las contrataciones de profesionales latinoamericanos, o europeos vinculados al continente, fue una práctica cada vez mayor en el organismo de Ginebra, seguida muy de cerca por su propio director Albert Thomas. Los casos de Moisés Poblete, Antonio Fabra Rivas, Carlos García Palacios (YÁÑEZ, 2013; 2014; FERRERAS, 2019), por citar algunos ejemplos, representaron el esfuerzo de la OIT por avanzar en una agenda que garantizara sus principios y acciones como organización universal y no primordialmente europea. 


\section{Distintas escalas de una relación: La OIT y América del Sur}

En los últimos años los estudios transnacionales han tenido un importante desarrollo, ofreciendo miradas renovadoras sobre los procesos regionales, las instituciones internacionales y los actores que participan en el dominio supranacional (LINDEN, 2006; IRIYE; SAUNIER, 2009). Los estudios sobre las organizaciones internacionales, tradicionalmente enmarcados en la perspectiva de la historia diplomática y de las relaciones internacionales, tienen una importancia fundamental en la formación del mundo contemporáneo, tal como lo señala el historiador Akira Iriye (2002). Si los estudios sobre la OIT han comprendido un largo conjunto de temáticas y perspectivas historiográficas, las investigaciones actuales que han adoptado el enfoque transnacional obligan a poner el acento en los procesos de internacionalización de una organización que tradicionalmente fue vista como "internacional" a partir de la sola constatación de su declaración de principios (SEEKINGS, 2010; KOTT, 2008; 2011; SAUNIER, 2008; MAUL, 2017; 2019; BORIS, 2019). Este cambio de mirada obliga a desplazarse en relación con el marco europeo tradicional de investigación sobre la institución de Ginebra, con el fin de comprender los costos humanos y materiales de esta internacionalización hacia una región del mundo muy diferente de Europa. Sin embargo, este esfuerzo de renovación de los estudios sobre la OIT, desde la propia Oficina en Ginebra o centros de pensamiento europeo no ha involucrado a América Latina, siendo muy limitados las referencias en trabajos colectivos o individuales (LUCASSEN, 2006; VAN DAELE, 2010; LESPINET-MORET; VIET, 2011; KOTT; DROUX, 2013; MAUL, 2019; BORIS, 2019).

Para el caso de América Latina los trabajos sobre la OIT siguen siendo escasos, aunque en las últimas décadas se observa un mayor interés por parte de historiadoras/es y cientistas sociales a tratar la temática. Los primeros estudios apuntaron a reconocer las iniciales relaciones entre la institución de Ginebra y los países del continente, particularmente en materia de incorporación a la OIT y ratificación de las convenciones internacionales (GONZÁLEZ, 1986; YÁÑEZ, 2000; JACOB, 2005; HERRERA LEÓN, 2011). Los vínculos entre el director de la OIT, Albert Thomas, y algunos gobiernos latinoamericanos, han permitido dimensionar los alcances de la diplomacia ginebrina, incluso incorporando el rol de la Sociedad de Naciones y el panamericanismo (HERRERA LEÓN, 2011; FERRERAS, 2011; MCPHERSON; WEHRLI, 2015; YÁÑEZ, 2016; HERRERA LEÓN; WEHRLI, 2019). Recientemente tres importantes estudios han ofrecido las primeras aproximaciones sobre la presencia de la OIT en el continente. Primero, el trabajo de coordinación que llevaron a cabo los historiadores Patricio Herrera y Fabián Herrera (2013), el cual aborda aspectos referidos al desarrollo de la burocracia ginebrina, los vínculos de la OIT con intelectuales latinoamericanos que actuaron como corresponsales, los programas de asistencia técnica y la Primera Conferencia Interamericana del Trabajo llevada a cabo en Santiago de Chile en 1936. Luego, desde un enfoque que busca ofrecer una mirada regional de la OIT, Laura Caruso y Andrés Stagnaro (2017) recopilaron trabajos que destacan los avances en materia legislativa y las instancias de difusión de las nuevas normas laborales. Recientemente, un tercer trabajo colectivo continúa ampliando y profundizando algunos de estos registros para Argentina y Brasil, compilación coordinada por Norberto Ferreras, Andrés Stagnaro y Laura Caruso (2018). Por su parte, la historiadora argentina Laura Caruso (2016) abordó los vínculos entre el movimiento de trabajadores marítimos argentinos y las convenciones de la OIT y Juan Carlos Yáñez (2016) estudió en profundidad la relación que estableció Chile con la institución de Ginebra, en especial 
la preocupación manifestada por Albert Thomas frente a la creciente presencia del comunismo en las organizaciones obreras. Patricio Herrera González (2013b; 2013c; 2018a; 2018b) ha estudiado la relación entre la Confederación de Trabajadores de América Latina (CTAL) y su presidente, el dirigente sindical mexicano Vicente Lombardo Toledano, y la OIT, destacando la necesidad que tenía esta última en articular un vínculo más estrecho y permanente con una organización sindical de alcance continental.

Una dimensión que no se ha abordado lo suficiente en la historiografía latinoamericana, dice relación con la importancia que tuvo la OIT en la circulación de experiencias y conocimientos en materia de legislación laboral, sindicalización y conciliación, en especial en el Cono Sur de América (FERRERAS, 2013; 2019) aunque existen dos estudios sobre la experiencia de cooperación técnica que desarrolló la OIT en Venezuela (PLATA, 2013; YÁÑEZ, 2017). Esto explica, en parte, el sesgo que ha existido en la crítica expresada por los historiadores del movimiento obrero (HERRERA GONZÁLEZ, 2013b; 2009) hacia la temprana legislación social, o los cuestionamientos a los reales alcances de las oficinas o departamentos del trabajo y el de los funcionarios gubernamentales o internacionales, como por ejemplo el caso de Moisés Poblete (Chile), Alejandro Unsain (Argentina), Afonso Bandeira de Mello (Brasil) y José Enrique Sandoval y Saavedra (Cuba), solo por mencionar a algunos.

En un momento previo, las historiografías nacionales, políticas y obreras, han reproducido a lo largo de las décadas una perspectiva lineal y mecanicista sobre el desarrollo de la institucionalidad laboral, con prefiguradas concepciones sobre el rol del Estado, la burocracia, la legislación, el gremio empresarial o el sindicalismo obrero (ALEXANDER, 2009; ALBA, 1964; GODIO, 1985; GONZÁLEZ CASANOVA, 1984; KOVAL, 1985; BERGQUIST, 1988; MELGAR, 1988; ROXBOROUGH, 1997; ANGELL, 1997; KOFAS, 1992). Sin desconocer las aportaciones de las historias nacionales o regionales, esta contribución pone el acento en una escala continental e internacional, existiendo evidencias para reconocer el papel que tuvo la OIT en establecer relaciones permanentes con la clase política, técnicos, legisladores, empresarios y dirigentes obreros, instaurando un espacio de convergencia y debate, el cual no estuvo exento de intransigencia y defensas ideológicas, pero que permitió avanzar hacia una sociedad del trabajo. Si bien los diversos actores latinoamericanos tenían una agencia propia, la relación con misiones técnicas y directores de la OIT en diferentes reuniones oficiales, en Ginebra o en ciudades americanas, potenció los propios espacios de encuentro y la legitimación de los múltiples protagonistas. Este proceso supuso dotar de saberes técnicos a todos los involucrados, cuestión que aún presenta opacidades desde la historiografía y que esperamos poder avanzar en próximos estudios para ofrecer un panorama más integral sobre aspectos tan relevantes como: la cientificidad del trabajo; la seguridad social; la modernización de las relaciones laborales: la participación del sindicalismo en el debate de la institucionalidad laboral; la negociación con los empresarios sobre condiciones de bienestar. Todos aspectos que están ampliando el conocimiento sobre la región y la OIT, en una colaboración regional y continental entre historiadoras/es del Cono Sur (BASUALDO, 2010; HERRERA GONZÁLEZ, 2013a; 2013c; 2015; REMEDI, 2014; RAMACCIOTTI, 2015; SCODELLER, 2016; YÁÑEZ, 2014; 2016; VIDELA, 2016; VERGARA, 2018).

La OIT no puede pensarse históricamente como una institución supranacional que solo propuso un dialogo tripartito, transfirió normas y procedimientos del trabajo o alentó la aprobación de resoluciones con los Estados miembros. Al contrario, su relación con los distintos actores 
latinoamericanos le permitió al organismo ginebrino deseuropeizar su "misión universalista", conocer nuevos problemas continentales y regionales en América Latina (YÁÑEZ, 2014). Como resultado de ello hubo una interacción entre la OIT y en especial con los países del Cono Sur de América (Brasil, Uruguay, Argentina y Chile), sobre la base de la cooperación técnica, redes políticas y negociaciones sindicales, particularmente fue fructifero el periodo entre 1936-1964. En el caso de los países andinos (Ecuador, Perú y Bolivia) las formas extendidas de trabajo campesino, indígena o esclavista promovió estudios y debates particulares, pues era evidente la dicotomía que producía el derecho y las resoluciones sancionadas por la OIT. Es lo que Rossana Barragán (2017) ha denominado como la geografía diferencial de los derechos.

El papel de los funcionarios de la OIT y de los incipientes Departamentos o Ministerios del Trabajo fue crucial a la hora de promover una mayor circulación de los saberes y de reunión de instancias técnicas y de debates. A partir de los años 1940, funcionarios ginebrinos, pero también de origen latinoamericano, como Moisés Poblete, Alejandro Unsain, Nicolás Repetto, Rafael Caldera, Bandeira de Mello, Saavedra Lamas, tuvieron un rol preponderante, hicieron circular sus estudios sociales, expusieron sus puntos de vista en conferencias internacionales del trabajo, produjeron intercambios de información o prestaron sus asesorías a gobiernos (HERRERA GONZÁLEZ, 2013a; 2013b; YÁÑEZ, 2013; 2014; 2018; RAMACCIOTTI, 2015; CARUSO; STAGNARO, 2017).

Por tanto, es relevante en el marco de una agenda de investigación nacional y continental avanzar en el análisis de los crecientes vínculos regionales e internacionales entre múltiples actores, dirigentes y organizaciones sindicales, funcionarios, legisladores, políticos, profesionales-técnicos y organizaciones patronales, y cómo estos vínculos permitieron o no avanzar en un mejoramiento de la institucionalidad laboral, dado que los contextos de guerra, postguerra y hegemonías contribuyeron en algunos momentos a fortalecer y en otros a debilitar los idearios de trabajadores y empresarios, y a los necesarios reacomodos de la burocracia estatal.

Durante la década de 1930 el escenario laboral del continente comienza a sufrir modificaciones que dieron forma a una nueva cultura del trabajo (YÁNEZ, 2008; BEN PLOTKIN; ZIMMERMAN, 2012; HERRERA GONZÁLEZ, 2013a; DRINOT, 2016), obligando a una modernización en las funciones del Estado, a definir derechos y deberes en las relaciones laborales y establecer mayores acercamientos con las organizaciones de la clase trabajadora. Los nuevos mecanismos de control laboral; el incremento de la sociedad salarial; las agencias, públicas y privadas, ocupadas de asesorar técnicamente a los gobiernos, empresarios y trabajadores con el objetivo de optimizar los recursos humanos y económicos; los Estados, junto a obreros y patrones, debatiendo sobre las coberturas de los accidentes del trabajo o la seguridad social. Todos procesos que son necesarios revisitarlos desde la investigación histórica y las ciencias sociales, en una relación que permita ampliar las experiencias, explicaciones e interpretaciones, particularmente del Cono Sur de América por sus mayores vículos que logró establecer con la institucionalidad laboral emanda de la oficina de Ginebra.

La crisis de 1929 y la consiguiente Gran Depresión (DRINOT; KNIGHT, 2015) fue un parteaguas entre las primeras relaciones que la OIT había establecido con América Latina y la etapa que se comenzó a desarrollar a partir de la década de 1930. La crisis de 1929, con sus efectos en la cesantía y producción, relanzó la agenda social de distintos organismos y reuniones panamericanas, cambiando el paradigma económico vigente hasta ese momento (MADDISSON, 1988). 
Frente a la crisis económica, y sus efectos inesperados en el comercio mundial, la OIT asumió desde el comienzo una posición clara, aunque no exenta de dilemas. La crisis de 1929 y sus efectos era la prueba de la necesidad de integrar al estudio de las políticas sociales la dimensión económica. Aunque después de su creación la OIT buscó ampliar su dominio de acción, incorporando en la discusión temas como la organización científica del trabajo o los aspectos financieros, la crisis de 1929 obligó a un esfuerzo de coordinación entre las diversas instituciones y países como jamás se había visto, cuestionando su capacidad de enfrentar la crisis con las herramientas tradicionales: el sistema de recomendación y de convención. El funcionamiento de las conferencias internacionales le daba un peso específico al resultado del debate y posterior acuerdo del tripartidismo -Estado, trabajadores, empresarios -, sin embargo los Estados latinoamericanos en la práctica hacían inoperante la ratificaciones de la convenciones, ya que tenían una inspección débil y alianzas políticas interesadas en mantener el statu quo, cuestión que se modificó con la mayor presencia efectiva e interacción de la OIT y sus interlocutores regionales en suelo americano.

En este escenario la OIT convocó a la Primera Conferencia americana del trabajo de los países miembros de la OIT en 1936, lo que permitió una oportunidad histórica para consolidar la institucionalidad laboral en el Cono Sur de América (HERRERA GONZÁLEZ, 2013a; 2015; 2017; QUEIROLO, 2017; RAMACCIOTTI, 2015; 2017; YÁÑEZ, 2014). Entre 1936 y 1961, la OIT en alianza con los Estados, organizaciones sindicales y patronales, organizó importantes conferencias regionales del trabajo en América Latina, (Santiago de Chile, 1936; La Habana, 1939; Ciudad de México, 1946; Montevideo, 1949; Río de Janeiro, 1952; La Habana, 1956; Buenos Aires, 1961) con el objetivo de aproximar los preceptos y proposiciones del organismo ginebrino a suelo americano, cuestión que varios trabajos de este recuento han complejizado en el presente, superando las visiones neutras o acríticas que hubo sobre estas conferencias y reuniones oficiales. En cada una de ellas los delegados gubernamentales, patronales y sindicales, debatieron el informe del director de la OIT, logrando importantes resoluciones en materia económica, social y laboral para todas las partes involucradas (HERRERA GONZÁLEZ, 2015; 2018a). Esto se tradujo en una oportunidad para avanzar hacia una conquista de derechos sociales y económicos para la clase trabajadora, un mejoramiento en la productividad para los empresarios y un compromiso para favorecer el desarrollo social para el Estado. En síntesis, la asistencia técnica de la OIT - a través de las Conferencias americanas del trabajo - generó un espacio de debate, entre las oportunidades y límites que tenía el Cono Sur para alcanzar mejores estándares de integración económica y bienestar social. El derecho y la legislación sociolaboral fueron una vía reformista, aunque no se excluyó el discurso de clase en las organizaciones sindicales, en medio de un escenario internacional complejo y de nutridos conflictos geopolíticos y económicos. El derecho garantizaba avanzar en la institucionalidad social, financiada y programada por el Estado, que con el tiempo fue otorgando mayores prestaciones para la clase trabajadora, aún en periodos de crisis. (HERRERA GONZÁLEZ, 2018a; VERGARA, 2018; VIDELA, 2016; DRINOT; KNIGTH, 2015; REMEDI, 2014; JENSEN, 2011).

Las décadas de 1940, 1950 y 1960, son particularmente complejas, pues el fin de la Segunda Guerra Mundial, el inicio de la Guerra Fría y el reacomodo de las hegemonías ideológicas del capitalismo y comunismo, expresadas por ejemplo en la Revolución Cubana y el derrocamiento de João Goulart, son el contexto más global para desentrañar el protagonismo que va adquiriendo la instalación de una sociedad laboral, en un contexto de creciente intervención social de raíces nacionales y transnacionales. 
Los diversos estudios nacionales y regionales que se han desarrollado en la última década - y que forman parte de este recuento - han indentificado las acciones técnicas, políticas y sindicales que desarrollaron la OIT y las organizaciones gubernamentales, patronales y sindicales entre 19361976 en el Cono Sur de América, incorporando una perspectiva comparada y transnacional, una cuestión que ya comienza a consolidar grupos de estudios, publicaciones, talleres y redes regionales de investigadoras/es. Esta historiografía, que está ganando en volumen e institucionalización dentro del campo de estudios sobre la OIT, ha tenido mayores impactos en el continente pero aún falta una recepción y circulación de sus contribuciones en la propia oficina con asiento en Ginebra y en producciones académicas de los países europeos.

\section{Rol de la OIT en la Guerra Fría latinoamericana: entre cooperación y "power soft"}

Un actor relevante de la historia laboral mundial, y particularmente latinoamericana, es el sindicalismo. El aporte de las historiografías obreras nacionales para interpretar los repertorios de organización y lucha fueron sustanciales en las investigaciones desarrolladas entre los años 1940 y 1990 (POBLETE, 1946; ALEXANDER, 1947; 1965; 2009; ALBA, 1964; GODIO, 1985; GONZÁLEZ CASANOVA, 1984; BERGQUIST, 1988; MELGAR, 1988; KOFAS, 1992). Las cronologías, las apropiaciones y conexiones con corrientes extranjeras, las tendencias políticas, los (des)encuentros con la clase política y gobernante, las confrontaciones con la clase patronal y la formación de su identidad como clase social, entre muchos otros tópicos, han ofrecido un conocimiento riguroso en datos, explicaciones y análisis. Sin embargo, como resultado de un acentuado contexto histórico de antagonismos, desplegado durante la Guerra Fría, hay que puntualizar que las historiografías obreras se interpretaron considerando los binomios dependencia-desarrollismo, centro-periferia, capitalismo-comunismo, lo que produjo una opacidad sobre numerosos hechos y procesos históricos (ALEXANDER, 2009; BETHELL, 1992; ROXBOROUGH, 1997; ANGELL, 1997; SPENSER, 2004).

La presencia de la OIT en el continente latinoamericano permitió abrir nuevos cauces, pero fue necesaria la voluntad de un movimiento de trabajadores y sus organizaciones dispuestas a negociar, la iniciativa de agencias obreras o gubernamentales que realizaran estudios técnicos sobre diversas materias laborales, económicas y sociales, tales como: el nivel de los salarios; la integración indígena; la situación laboral y social del campesinado; la seguridad social; la inserción de la medicina del trabajo; la educación técnica o la participación política de los trabajadores. En concreto, se trató de instaurar una agenda laboral y social que estaba plenamente vigente en el debate sindical, patronal y estatal, pues había problemas estructurales aún sin solución efectiva. Para responder a las demandas de la sociedad salarial (CASTEL, 1997) ${ }^{2}$ se requirió una articulación entre el Estado, la OIT, las organizaciones de patrones, el sindicalismo obrero internacional de la Confederación de Trabajadores de América Latina (CTAL), Federación Sindical Mundial(FSM), Confederación Internacional de Organizaciones Sindicales Libres (CIOSL), Organización Regional Interamericana del Trabajo (ORIT) y las organizaciones sindicales nacionales (BASUALDO, 2013; CORREA, 2017; HERRERA GONZÁLEZ, 2016; RODRÍGUEZ, 2010; SCODELLER, 2013; 2016; 2017; YÁÑEZ, 2017; ZORZOLI, 2018). 
Fue la Conferencia Americana del Trabajo, de 1936, la que inauguró una relación más colaborativa, que en retrospectiva le permitió a la OIT planificar su misión universalista con nuevos derroteros, orientando sus principios de acción, que luego se utilizaron para promover los derechos sociales y laborales en regiones fuera del espectro europeo, como fueron los casos de Asia y África en la década de 1960 (VAN DAELE, 2010; KOTT, 2013; MAUL, 2017).

Los derechos sociales formaron parte de las exigencias laborales de las organizaciones obreras, cuyas reivindicaciones se acrecentarían, tendrían un nuevo escenario, se configuraron de manera más compleja con la participación de la OIT en el Cono Sur de América. Los debates e informes técnicos, discutidos en cada Conferencia americana del trabajo conllevaron esfuerzos de implementación de sus resoluciones en políticas sociales concretas, muchas de las cuales fueron resultado de consensos entre la clase trabajadora y empresarial, allanando el camino para el reconocimiento del trabajador como sujeto de derecho, político, económico y cultural, lo que modificó sustancialmente su relación con los medios de la "propiedad social”, tal como lo identificó Robert Castel (1997) para el caso europeo.

La segunda posguerra modificó el panorama geopolítico mundial, donde el binomio capitalismo-comunismo se impuso con vehemencia en todos los continentes. Tanto el liderazgo de Estados Unidos como el de la Unión Soviética presionaron a sus periferias. Los hechos ocurridos en América Latina así lo confirman, en particular lo que sucedió con las organizaciones obreras organizadas y la constante inflitración que sufrieron sindicatos, partidos políticos e intelectuales vinculados a concepciones prosoviéticas o antinorteamericanas, lo que significó quebrantar la unidad obrera conquistada y de paso cuestionar la capacidad de negociación política de los trabajadores. Recientes investigaciones proponen nuevas miradas con respecto a estos procesos, pues la presencia de la OIT no se había considerado en este escenario histórico bipolar (RODRÍGUEZ, 2010; BASUALDO, 2010; HERRERA GONZÁLEZ, 2016; 2018a; SCODELLER, 2013; 2016; 2017; CORREA, 2017; YÁÑEZ, 2017; ZORZOLI, 2018).

La presencia del organismo ginebrino en América Latina se movió desde apoyos a la autonomía y libertad sindical, a la promoción de escuelas sindicales anticomunistas, que junto a la colaboración - financiera, logística e ideológica - de la American Federation of Labor (AFL)/Congress of Industrial Organizations (CIO) proprocionaron las bases de un nuevo sindicalismo, cuestión que estudios recientes están comenzado a determinar y analizar. Investigaciones han avanzado en establecer la inflitración que realizó la AFL/CIO en la CTAL, donde en un principio la OIT tuvo un rol de enlace entre funcionarios, dirigentes y organizaciones para defender la autonomía y libertad sindical (HERRERA GONZÁLEZ, 2016). También, hoy conocemos la importancia de la creación de la Confederación Internacional de Organizaciones Sindicales Libres (CIOSL), fundada en 1949, y la Organización Regional Interamericana de Trabajadores (ORIT), inaugurada en 1951. Ambas organizaciones fueron financiadas y apoyadas por la AFL/CIO, con una presencia importante en América Latina, entre 1949 y 1970. Estas plataformas sindicales, internacional y regional, reclutaron a organizaciones laborales desprovistas de ideologías uniformes, donde el apoyo de Estados Unidos en lo económico y formativo fue primordial para inclinar sus motivaciones anticomunistas en el continente (RODRÍGUEZ, 2010).

Recientemente, se han desarrollado estudios que contribuyen a comprender el rol de institutos de formación sindical creados en los años sesentas, como parte de la escalada norteamericana ante el triunfo de la Revolución Cubana.Tal es el caso de el Instituto Internacional de Estudios Laborales 
(IIEL), creado por la OIT; el Instituto Interamericano de Estudios Sindicales (IIES), fundado por la ORIT; el Instituto Americano para el Desarrollo del Sindicalsmo Libre (IADSL), patrocinado por la AFL/CIO; y el Instituto Latinoamericano de Planificación conómica y Social (ILPES), creado por la CEPAL. Todos ellos contribuyeron a movilizar un sindicalismo con capacidades técnicas, de planificación económica-social y liderazgos de ascendencia internacional-regional de influencia pronorteamericana (SCODELLER, 2016; 2017; CORREA, 2017). Todas cuestiones que necesitan ser ampliadas y analizadas, para comprender el rol de la OIT que paulatinamente se transformó en un "power soft", pues su influencia en sectores sindicales y gremiales de América Latina se fue atomizando, dada la misma fragmentación de las organizaciones y su polarización ideológica, como resultado de la Guerra Fría, y donde la propia OIT quedó atrapada en propósitos laborales y legislativos con menos resultados sobre amplios sectores de trabajadores, en el mejor de los casos, o fue un espectador neutro y acrítico de la influencia de Estados Unidos en la región.

\section{A manera de cierre (abierto)}

Este artículo ha relevado las temáticas de las investigaciones que están realizando historiadoras e historiadores, cientistas sociales, latinoamericanos y latinoamericanistas, al incorporar las experiencias históricas que se conservan en los archivos de la Oficina Internacional del Trabajo, en Ginebra, como también en acervos y archivos locales que nos permite reconstruir e interpretar el vínculo de la OIT y la región, aunque con ciertas limitaciones. Todo ello ha posibilitado decodificar otras claves políticas, legislativas, sociales y técnicas de la historia laboral del continente. Se consolidó una agenda historiográfica de largo alcance, pues las épocas, temáticas, protagonistas e interpretaciones suponen avanzar en nuevos conocimientos sobre nuestra historia, particularmente del Cono Sur, en clave regional, con una nueva perspectiva, una nueva escala, y nuevas preguntas que surgen de los vínculos y mutuas determinaciones. Temas de género, infancia, programas de bienestar del empresariado, saberes del Estado, programas sanitarios, son cuestiones que seguramente serán las próximas contribuciones por venir.

Recientemente aparecieron algunas obras colectivas (WEINBERG, 2019) o de síntesis (FERRERAS, 2019; VILLASMIL, 2019), a propósito del centenerio de la OIT, lo que viene a reafirmar que hay un campo de saberes que colabora en complejizar y trascender nuestras historias nacionales, desde la mirada continental. A contracorriente de la propia OIT y sus historiadores oficiales del centenario (MAUL, 2019; BORIS, 2019) que siguen reconstruyendo una historia con mirada eurocéntrica, esta contribución presenta un recuento de una profusa e importante investigación, de numerosas historiadoras e historiadores y cientistas sociales, que con numerosas evidencias de archivos, folletos, hemerografía, periódicos, oficios, correspondencia y memorias proporcionan interpretaciones y relaciones entre América Latina y la OIT, demostrando que la región y sus interlocutores - políticos, legisladores, funcionarios, dirigentes obreros, dirigentes gremiales, empresarios - le ofrecieron a la organización ginebrina la posibilidad de crear un espacio de presencia y legitimación, no para cumplir su misión universal de justicial social y paz universal, pero sí para sostener la verosimilitud de esta. 


\section{Referencias}

ALBA, Víctor. Historia del Movimiento Obrero en América Latina. México: Libreros Mexicanos Unidos, 1964.

ALEXANDER, Robert J. Labour Movements in Latin America. London: Fabian Publications, 1947.

ALEXANDER, Robert J. Organized Labor in Latin America. New York: Free Press, 1965.

ALEXANDER, Robert J. International Labor Organizations and Organized Labor in Latin America and the Caribbean; A History. Santa Barbara: Praeger/ABC-CLIO, 2009.

ANGELL, Alan. La izquierda en América Latina desde 1920, en Leslie Bethell (Ed.). Historia de América Latina. tomo 12. Barcelona: Cambridge University Press y Crítica, 1997.

BARRAGÁN, Rossana. La geografía diferencial de los derechos: entre la regulación del trabajo forzado en los países coloniales y la disociación entre trabajadores e indígenas en los Andes (1920-1954). In: CARUSO, Laura; STAGNARO, Andrés. Regular y Legislar el mundo del trabajo Latinoamericano. Aportes para una historia regional de la OIT. La Plata, 2017.

BASUALDO, Victoria. The ILO and the Argentine dictatorship, 1976-1983. In: Van DAELE, Jasmien et al. (Eds.). ILO Histories: Essays on the International Labour Organization and Its Impact on the World During the Twentieth Century. Bern: Peter Lang AG, International Academic Publishers, 2010.

BASUALDO, Victoria. El movimiento sindical argentino y sus relaciones internacionales: una contribución sobre la presencia de la CIOSL y la ORIT en la Argentina desde fines de los ${ }^{-} 40$ hasta comienzos de los ${ }^{-} 80$. In: Mundos do Trabalho, 2013.

BERGQUIST, Charles. Los trabajadores en la historia latinoamericana. Bogotá: Siglo XXI editores, 1988.

BETHELL, Leslie; ROXBOROUGH, Ian. Latin America between second world war and the cold war - 19441948. New York: Cambridge University Press, 1992.

BORIS, Eileen. Making the woman worker. New York: Oxford University Press, 2019.

CARUSO, Laura. Embarcados. Los trabajadores marítimos y la vida a bordo: sindicato, empresas y Estado en el puerto de Buenos Aires, 1889-1921. Buenos Aires: Imago Mundi, 2016.

CARUSO, Laura; STAGNARO, Andrés. Regulary Legislar el mundo del trabajo Latinoamericano. Aportes para una historia regional de la OIT. La Plata, 2017.

CASTEL, Robert. La metamorfosis de la cuestión social. Buenos Aires: Paidós, 1997.

CORREA, Larissa. Disseram que voltei americanizado: relações sindicais Brasil-Estados Unidos na ditadura militar. Brasil: Editora UNICAMP, 2017.

DRINOT, Paulo; KNIGHT, Alan. La Gran Depresión en América Latina. México: Fondo de Cultura Económica, 2015.

DRINOT, Paulo. La seducción de la clase obrera: trabajadores, raza y la formación del Estado peruano. Traducción de Óscar Hidalgo W. Lima: IEP: Ministerio de Cultura, 2016.

FERRERAS, Norberto. La OIT y los países del Cono Sur en el período de entreguerras. Montevideo: Fundación Electra: Fundación de Cultura Universitaria, 2019.

FERRERAS, Norberto; STAGNARO, Andrés; CARUSO, Laura. A Conexão OIT. América Latina: Problemas regionais do trabalho em perspectiva transnacional. Niteroi: Editora Mauad, 2018.

FERRERAS, Norberto. Entre a expansão e a sobrevivência: a viagem de Albert Thomas ao Cone Sul da América. In: Antíteses. n. 7, enero-junio 2011. p. 127-150.

GODIO, Julio. Historia del movimiento obrero latinoamericano. tomo III. San José: Editorial Nueva Sociedad,1985.

GONZÁLEZ CASANOVA, Pablo (coord.). Historia del movimiento obrero en América Latina. 4 tomos. México: Instituto de Investigaciones Sociales de la UNAM: Siglo XXI Editores, 1984.

GONZÁLEZ, Aída. México y la Organización Internacional del Trabajo. In: México y la paz. México: Instituto Matías Romero de Estudios Diplomáticos-SRE, 1986. 
HERRERA GONZÁLEZ, Patricio. Trabajo, industrialización y justicia social. La clase obrera organizada de America Latina y su programa continental para la posguerra (1939-1948). In: SIMONASSI, Silvia; DICÓSIMO, Daniel (comp.). Trabajadores y sindicatos en Latinoamérica: conceptos, problemas y escalas de análisis. Buenos Aires: Imago Mundi, 2018a.

HERRERA GONZÁLEZ, Patricio. Colaboraciones técnicas y políticas trasatlánticas: América Latina y la OIT (1928-1946). Estudios Internacionales. v. 189, 2018b. p. 77-96.

HERRERA GONZÁLEZ, Patricio (coord.). El Comunismo en América Latina: experiencias militantes, intelectuales y transnacionales (1917-1955). Valparaíso: Universidad de Valparaíso, 2017.

HERRERA GONZÁLEZ, Patricio. El asedio a la Clase Obrera Organizada en los inicios de la Guerra Fría: el caso de la CTAL - 1943-1953. Divergencia, v. 6, 2016. p. 29-39.

HERRERA GONZÁLEZ, Patricio. Las Conferencias Americanas del Trabajo y el debate sobre las condiciones laborales del proletariado de América Latina - 1936-1946. Mundos do Trabalho. v. 13, 2015. p. 105-128.

HERRERA GONZÁLEZ, Patricio. La primera conferencia regional del trabajo en América: su influencia en el movimiento obrero, 1936. In: HERRERA, Fabián; HERRERA, Patricio (coords.). América Latina y La Organización Internacional del Trabajo. Redes, cooperación técnica e institucionalidad social, 1919-1950. Morelia: Instituto de Investigaciones Históricas: Universidad Michoacana de San Nicolás de Hidalgo, $2013 a$.

HERRERA GONZÁLEZ, Patricio. La Confederación de Trabajadores de América Latina. Una historia por (re) significar - 1938-1963. Secuencia, n. 86, Instituto de Investigaciones Mora, mayo-agosto 2013b. p. 195-218.

HERRERA GONZÁLEZ, Patricio. La Confederación de Trabajadores de América Latina y la implementación de su proyecto sindical continental - 1938-1941. Trashumante. Revista Americana de Historia Social, v. 2 , 2013c. p. 136-164.

HERRERA GONZÁLEZ, Patricio (Reseña); ANDRADE, Juan Carlos Yáñez. La intervención social en Chile, 1907-1932. Relaciones. Estudios de historia y sociedad, v. 30, n. 119, 2009. p. 227-233.

HERRERA LEÓN, Fabián; WEHRLI, Yannick (coords.). América Latina y el internacionalismo ginebrino de entreguerras: implicancias y resonancias. México: Dirección General del Acervo Histórico Diplomático de la Secretaría de Relaciones Exteriores, 2019.

HERRERA LEÓN, Fabián; HERRERA GONZÁLEZ, Patricio (coords.). América Latina y la Organización Internacional del Trabajo. Redes, cooperación técnica e institucionalidad social - 1919-1950. Morelia: Instituto de Investigaciones Históricas: Universidad Michoacana de San Nicolás de Hidalgo, 2013.

HERRERA LEÓN, Fabián. México y la Organización Internacional del Trabajo: los orígenes de una relación, 1919-1931. In: Foro Internacional, n. 2, 2011.

HERRERA LEÓN, Fabián; WEHRLI, Yannick. Le Bureau international du travail et l'Amérique latine durant l'entre-deux-guerres. In: LESPINET-MORET, Isabelle; VIET, Vincent (coords.). L'Organisation internationale du travail. Origine, développement, avenir. Rennes: Presses Universitaires de Rennes, 2011.

IRIYE, Akira; SAUNIER, Pierre-Yves. The Palgrave Dictionary of Transnational History. New York: MacMillan Publisher, 2009.

IRIYE, Akira. Global Community. The Role of International Organizations in the Making of the Contemporary World. Berkeley; Los Angeles: University of California Press Ltd, 2002.

JACOB, Raúl. Tecnócratas, revolucionarios y reformistas: la OIT en Uruguay. In: Mosaico. Montevideo: Editorial Arpoador, 2005. p. 11-47.

JENSEN, Jill. From Geneva to the Americas: the International Labor Organization and Inter-American Social Security Standards - 1936-1948. In: International Labor and Working-Class History. n. 80, 2011.

KOFAS, Jon. The Struggle for Legitimacy: Latin American Labor and the United States, 1930-1960. Tempe: Arizona State University, 1992.

KOTT, Sandrine; DROUX, Joëlle. Globalizing Social Rights: the International Labour Organization and beyond. Palgrave, 2013. 
KOTT, Sandrine. Dynamiques de l'internationalisation: I'Allemagne et l'Organisation internationale du travail - 1919-1940. In: Critique internationale. n. 52, julio-sept. 2011. p. 69-84.

KOTT, Sandrine. Les organisations internationales, terrains d'étude de la globalisation. Jalons pour une approche socio-historique. In: Critique internationale, n. 3, v. 52, 2011. p. 9-16.

KOTT, Sandrine. Une communauté épistémique du social? Experts de l'OIT et internationalisation des politiques sociales dans l'entre-deux-guerres. In: Genèses, n. 2, v. 71, 2008. p. 26-46.

KOVAL, B. Movimiento obrero en América Latina - 1917-1959. Moscú: Editorial Progreso, 1985.

LESPINET-MORET, Isabelle; VIET, Vincent (coords.). L'Organisation internationale du travail. Rennes: Presses Universitaires de Rennes, 2011.

LINDEN, Marcel van der. Historia transnacional del trabajo. Valencia: Centro Francisco Tomás y Valiente UNED Alzira-Valencia, 2006.

LUCASSEN, Jan (ed.). Global Labour History: A State of the Art. Bern: Peter Lang AG: International Academic Publishers, 2006.

MADDISON, Angus. Dos crisis: América latina y Asia - 1929-1938 y 1973-1983. México: Fondo de Cultura Económica, 1988.

MAUL, Daniel. La Organización Internacional del Trabajo: 100 años de políticas sociales a escala mundial. Ginebra: Oficina Internacional del Trabajo, 2019.

MAUL, Daniel. Derechos humanos, desarrollo y descolonización: la Organización Internacional del Trabajo entre 1940-1970. Madrid: Plaza y Valdés Editores, 2017.

MCPHERSON, Alan; WEHRLI, Yannick (ed.). Beyond Geopolitics. New histories of Latin America at the League of Nations. Nuevo México: University of New Mexico Press, 2015.

MELGAR BAO, Ricardo. El movimiento obrero latinoamericano. Historia de una clase subalterna. Madrid: Alianza Editorial, 1988.

PLATA, Véronique. La difusión de las normas internacionales de trabajo en Venezuela (1936-1939): primer estudio sobre la práctica de la cooperación técnica internacional en la OIT. In: HERRERA, Fabián; HERRERA, Patricio (coords.). América Latina y la Organización Internacional del Trabajo. Redes, cooperación técnica e institucionalidad social, 1919-1950. Morelia: Instituto de Investigaciones Históricas: Universidad Michoacana de San Nicolás de Hidalgo, 2013.

PLOTKIN, Mariano; ZIMMERMANN, Eduardo (ed.). Los saberes del Estado. Buenos Aires: Edhasa, 2012.

POBLETE, Moisés. El movimiento obrero latinoamericano. México: Fondo de Cultura Económica, 1946.

QUEIROLO, Graciela. Igual salario por igual trabajo: la Organización Internacional del Trabajo y el Estado argentino frente al trabajo femenino (1919-1960). In: CARUSO, Laura; STAGNARO, Andrés. Regular y Legislar el mundo del trabajo Latinoamericano. Aportes para una historia regional de la OIT. La Plata, 2017.

RAMACCIOTTI, Karina. Diálogos transnacionales entre los saberes técnicos e institucionales en la legislación sobre accidentes de trabajo. Primera mitad del siglo XX. In: História, Ciências, Saúde-Manguinhos, v. 22, n. 1, 2015. p. 210-219.

RAMACCIOTTI, Karina. El Boletín Informativo de Leyes de Trabajo: Circulación de ideas y actores latino-americanos sobre políticas sociales (1942-1951). In: CARUSO, Laura; STAGNARO, Andrés. Regular y Legislar el mundo del trabajo Latinoamericano. Aportes para una historia regional de la OIT. La Plata, 2017.

REMEDI, Fernando; BARBOSA, Mario. Cuestión social, políticas sociales y construcción del estado social en América Latina. Córdoba: Siglo xx, 2014.

RODRÍGUEZ GARCÍA, Magaly. Liberal Workers of the World, Unite? The ICFTU and the Defence of Labour Liberalism in Europe and Latin America (1949-1969). Bern: Peter Lang, 2010.

RODRÍGUEZ GARCÍA, Magaly. Conclusion: the ILO's Impact on the World. In: VAN DAELE, Jasmien et al. (Eds.). ILO Histories: Essays on the International Labour Organization and Its Impact on the World During the Twentieth Century. Bern: Peter Lang, 2010. 
ROXBOROUGH, Ian. La clase trabajadora urbana y el movimiento obrero en América Latina desde 1930. In: BETHELL, Leslie (ed.). Historia de América Latina. tomo 12. Barcelona: Cambridge University Press y Crítica, 1997.

SAUNIER, Pierre-Yves. Trajectoires, projets et ingéniérie de la convergence et de la différence: les régimes circulatoires du domaine social 1800-1940. In: Genèses. Sciences sociales et histoire, p. 4-25, 2008.

SCODELLER, Gabriela. Desarrollo, participación sindical y circuitos transnacionales de formación durante los años sesenta y setenta: el caso de las dirigencias latinoamericanas de la ORIT-CIOSL. Iberoamericana. Berlin; Hamburgo; Madrid, 2017. p. 211-234.

SCODELLER, Gabriela. Political Training and Social Change in the 1960s and 1970s: The Educational Activities of the Latin American Central of Workers (CLAT), International Labor and Working-Class History, v. 90 , 2016. p. 93-110.

SCODELLER, Gabriela. (Des)encuentros en las experiencias de formación político-sindical en los años ‘60 en Argentina. Historia Crítica, 2013. p. 157-181.

SEEKINGS, Jeremy. The ILO and Welfare Reform in South Africa, Latin America, and the Caribbean, 19191950. In: VAN DAELE, Jasmien et al. (eds.). ILO Histories: Essays on the International Labour Organization and Its Impact on the World During the Twentieth Century. Bern: Peter Lang, 2010.

SPENSER, Daniela (coord.). Espejos de la Guerra Fría: México: América Central y el Caribe. Ciesas, 2004.

VAN DAELE, Jasmien et al. (eds.). ILO Histories: Essays on the International Labour Organization and Its Impact on the World During the Twentieth Century. Bern: Peter Lang AG, International Academic Publishers, 2010.

VAN DAELE, Jasmien et al. (eds.). Writing ILO histories: a State of the Art. In: VAN DAELE, Jasmien et al. (eds.). ILO Histories: Essays on the International Labour Organization and Its Impact on the World During the Twentieth Century. Bern: Peter Lang, 2010.

VERGARA, Ángela. Cuando los trabajadores no trabajan: una aproximación a la historia del desempleo en América Latina. In: SIMONASSI, Silvia; DICÓSIMO, Daniel (comp.). Trabajadores y sindicatos en Latinoamérica: conceptos, problemas y escalas de análisis. Buenos Aires: Imago Mundi, 2018.

VIDELA, Enzo; VENEGAS, Hernán; GODOY, Milton. El orden fabril. Paternalismo industrial en la minería chilena. Santiago: Ediciones América en Movimiento, 2016.

VILLASMIL, Humberto. Una visión “americana” del centenario de la OIT: aproximación a la comprensión de una relación histórica. Santiago: Oficina de la OIT para el Cono Sur de América Latina, 2019.

WEINBERG, Pedro Daniel (comp.). La OIT en América Latina. Montevideo: Fundación Electra, 2019.

YÁÑEZ ANDRADE, Juan Carlos. Moisés Poblete Troncoso y su aporte a la recopilación de la legislación laboral. In: Revista de Estudios Histórico-Jurídico, n. XL, 2018. p. 371-390.

YÁÑEZ ANDRADE, Juan Carlos. La Organización Internacional del Trabajo y la libertad sindical en América Latina: el caso de Venezuela en 1949. Anuario del Instituto de Historia Argentina, v. 17, n. 1, 2017.

YÁÑEZ ANDRADE, Juan Carlos. La OIT en América del Sur. El comunismo y los trabajadores chilenos - 19221932. Santiago: Editorial UAH, 2016.

YÁÑEZ ANDRADE, Juan Carlos. L'OIT et l'Amérique Latine. La construction d'un laboratoire social régional 1919-1950. Paris: EHEES. Tesis (doctorado en Historia y Civilizaciones), 2014.

YÁÑEZ ANDRADE, Juan Carlos. La intervención social en Chile - 1907-1932, Santiago de Chile: Ril, 2008.

YÁÑEZ ANDRADE, Juan Carlos. Chile y la organización del trabajo - 1919-1925. Hacia una legislación social universal. Revista de estudios histórico-jurídicos, n. 22, 2000. p. 317-332.

ZORZOLI, Luciana, Las intervenciones a organizaciones sindicales durante la última dictadura militar argentina: un estudio cuantitativo. Desarrollo Económico. v. 57, n. 223, enero-abril 2018. p. 487-510. 


\section{Notas}

${ }^{1}$ Entre las publicaciones más relevantes de Moisés Poblete, en relación a su condición de alto funcionario de la OIT, podemos considerar: La legislación social de América Latina (Ginebra, OIT, 1928-1929), vol. 1-2; Ensayo de bibliografía social de los países hispanoamericanos (Santiago, La Nación, 1936); Problemas sociales y económicos de América Latina (Santiago, Universidad de Chile, 1936); Condiciones de vida y de trabajo de la población indígena del Perú (Ginebra, OIT, 1938); Conferencia internacional del trabajo de Nueva York: 27 de octubre-6 de noviembre de 1941 (Santiago, Imprenta Universitaria,1942); Evolución del Derecho Social en América (Santiago, Editorial Nascimento, 1942); El movimiento obrero latinoamericano (México, FCE, 1946); El derecho del trabajo y la seguridad social en Chile (Santiago, Editorial Jurídica, 1949).

${ }^{2}$ Robert Castel se refiere al proceso de la sociedad salarial como la transformación que sufren las identidades de los trabajadores al extenderse el salario. Cuestión que supone instalar posiciones, diferencias, escalas, pero también promociones, progresos y protecciones, muchas de un carácter inédito para las vidas de mujeres, hombres, infantes y ancianos. El Estado y su agencia, como las propias luchas de los movimientos de trabajadores cooperaron en garantizar un bienestar, ampliando derechos y garantías.

Recebido em: 21/03/2020

Aprovado em: 10/06/2020 